\title{
Dust sedimentation in protoplanetary disks with polycyclic aromatic hydrocarbons
}

\author{
C. P. Dullemond ${ }^{1}$, Th. Henning ${ }^{1}$, R. Visser ${ }^{2}$, V. C. Geers ${ }^{2}$, E. F. van Dishoeck ${ }^{2}$, and K. M. Pontoppidan ${ }^{3}$ \\ 1 Max-Planck-Institute für Astronomie, Königstuhl 17, 69117 Heidelberg, Germany \\ e-mail: dullemon@mpia.de \\ ${ }^{2}$ Leiden Observatory, Leiden University, PO Box 9513, 2300 RA Leiden, The Netherlands \\ 3 California Institute of Technology, Division of Geological and Planetary Sciences, MC 150-21, Pasadena, CA 91125, USA
}

Received 1 April 2007 / Accepted 28 June 2007

\begin{abstract}
Context. Dust sedimentation is known to affect the infrared spectra and images of disks. In particular the far-infrared emission may be reduced by strong sedimentation. However, dust grains of different sizes sediment to different depths in the disk. Spectral features of one species may thus be enhanced, while those of other species may be suppressed.

Aims. Polycyclic aromatic hydrocarbons (PAHs) are among the smallest "dust grains". We investigate how the presence of PAHs in protoplanetary disks affects the disk's spectral energy distribution (SED) and feature strengths when the thermal (large) grains are allowed to sediment.

Methods. We calculate the vertical distribution of dust grains for both the PAH "dust" and the thermal dust grains. We include vertical settling and vertical mixing via turbulence. The results are inserted into a Monte-Carlo radiative transfer code to compute the SEDs. Results. For high turbulence the sedimentation barely affects the spectrum. For low turbulence, however, the PAHs still stay wellmixed in the disk's surface layer, while the $0.1 \mu \mathrm{m}$ size grains sediment deep into the disk. This strongly enhances the PAH features relative to the continuum (by factors of 2 to 10), while the far-infrared flux is reduced. This predicts that sources with weak farinfrared flux have stronger PAH features, which is - at least among Herbig Ae stars - opposite to what is observed, suggesting that sedimentation is not the only factor responsible for the weak mid- to far-infrared excess in some disks. We speculate that coagulation might be a solution, reducing both the mid- to far-infrared flux and the PAH features.
\end{abstract}

Key words. accretion, accretion disks - circumstellar matter - stars: formation - stars: pre-main-sequence - infrared: stars

\section{Introduction}

Polycyclic aromatic hydrocarbons (PAHs) are a well-known component of interstellar material. Considered to be extremely small dust grains by some, and very large molecules by others, they are made of a few tens to hundreds of carbon atoms. They are observed in the interstellar medium through their narrow emission features at 3.3, 6.2, 7.7, 8.6, 11.3 and $12.7 \mu \mathrm{m}(\mathrm{Li}$ $\&$ Draine 2001). These molecules are typically large enough that the excitation state of their internal vibrational modes can be reasonably well described by a temperature at any given time, but they are still so small that this temperature fluctuates in time for each individual PAH particle. A single ultraviolet (UV) photon can excite the particle to a state corresponding to a temperature of hundreds of Kelvins, after which it rapidly cools down until it is excited by the next UV photon. This allows PAHs to radiate at near- and mid-infrared wavelengths even at large distances from the stars from which these UV/optical photons originate. PAH emission is therefore often observed as diffuse emission at the edges of molecular clouds (e.g. Abergel et al. 1996; Churchwell et al. 2004). Recently, however, evidence has appeared that PAH emission can also originate from protoplanetary disks. It is mostly detected in disks around Herbig $\mathrm{Ae} / \mathrm{Be}$ stars (van Boekel et al. 2004; Habart et al. 2004, henceforth H04; Perrin et al. 2006) but also recently in disks around T Tauri stars (Geers et al. 2006, henceforth G06; Bouwman et al. submitted). This opens up various interesting possibilities to probe the physics of these disks. First, spatially resolved imaging in $\mathrm{PAH}$ features allows us to map the shape of the surface of the disk (Habart et al. 2006; Lagage et al. 2006), and it is confirmed that these disk surfaces are flared, as predicted from theory (Kenyon \& Hartmann 1987). Secondly, the presence of PAHs reveals that in these objects the dust coagulation process was apparently not effective enough to remove the PAHs from these disks, or that some other process continuously releases PAHs out of aggregates or from dust surfaces. Thirdly, the comparison of spectra and images in the PAH bands and in thermal dust emission reveals information about the sedimentation of grains in the disk. In this paper we will focus on this last topic.

Solid particles tend to sediment toward the equatorial plane of the disk as a result of the vertical component of gravity. While it may take a long time to sediment all the way to the midplane, the first stages of sedimentation can be orders of magnitude faster. Within a few hundred orbits, i.e. about $10^{5}$ years at $50 \mathrm{AU}$, the dust sediments down below a few pressure scale heights of a disk, effectively evacuating the upper layers of the disk (see e.g. Dullemond \& Dominik 2004b). Turbulence, however, tends to mix the grains back up again. The result is that sedimentation can proceed down to some height above the disk midplane below which the dust is well-mixed with the gas (e.g. Dubrulle et al. 1995; Schräpler \& Henning 2004). The dust thus forms a steady-state layer of a certain thickness, which for small enough grains can be several pressure scale heights thick, while for large grains (millimeter or more), or for low turbulence, it can drop below one scale height. 
Since dust is the main source of opacity, the sedimentation of dust down to its equilibrium thickness also affects the observed shape of the disk: the photosphere of the disk is dragged down with the dust. The spectral energy distribution (SED) of the disk is thereby also changed: typically the far-infrared (far-IR) part of the SED is suppressed (Miyake \& Nakagawa 1995; Dullemond \& Dominik 2004b, henceforth DD04b). It can be speculated that the different SED slopes in observed sources (Meeus et al. 2001; Furlan et al. 2006) may be related to this sedimentation. Whether sedimentation takes place in these disks remains speculative at this point, since very little observational evidence exists. An example of a direct measurement of the effects of sedimentation in disk is the work by Rettig et al. (2006) who measured column densities in dust and in gas (as measured by $\mathrm{CO}$ absorption) in four sources at varying near edge-on inclinations and concluded that there is evidence for dust depletion in the surface layers of these disks consistent with sedimentation and growth. Duchêne et al. (2003) derive evidence for sedimentation in one source (HK Tau B) based on detailed radiative transfer.

As we will show here, sedimentation tends to enhance the dust features originating from the smallest grains, since the larger grains sediment deeper into the disk than the smaller grains, hence allowing the smaller grains to dominate the photosphere. Since PAHs are the smallest "grains" imaginable, this effect is expected to be strongest for such particles, and we expect a strong enhancement of the PAH features originating in disks. The presence of other small grains that do not have these features, such as "Very Small Carbon Grains" (VSGs), would weaken this effect, as we will show. The strength of these PAH features depends, of course, also on the abundance of PAHs in the disk (G06). A weak observed PAH feature can therefore either mean that there is strong turbulence in the disk, or that the average PAH abundance is so low, that even with the effect of feature-enhancement through sedimentation the feature remains weak. On the other hand, very weak turbulence may affect the overall shape of the SED, as mentioned above, and hence any observed correlation between PAH feature strength and SED shape may provide clues to the level of turbulence and abundance of PAHs in disks. This is what we wish to investigate in this paper.

We present time-dependent models of the sedimentation of grains in a turbulent disk. Then we apply a multi-dimensional Monte Carlo radiative transfer tool to compute the spectrum at 1 Myr after the start of the simulation, in which a steady-state usually is reached. This follows the method outlined in DD04b.

\section{Model}

\subsection{Modeling method}

The modeling procedure consists of three steps. In the first step we construct a self-consistent passive disk model following the recipe of Dullemond \& Dominik (2004a, henceforth DD04a). This model involves 2-D radiative transfer coupled to the equation of vertical hydrostatic equilibrium. The main input parameters for this disk model are the parameters of the central star (mass $M_{*}$, radius $R_{*}$ and effective temperature $T_{*}$ ) and the surface density of the disk $\Sigma(R)$, where $R$ is the radial coordinate in the disk. We take as surface density profile a powerlaw profile $\Sigma(R) \propto R^{-1}$ with an inner and outer radius $\left(R_{\text {in }}\right.$ resp. $\left.R_{\text {out }}\right)$. The values we take for these parameters are described in Sect. 2.2. In this first step we assume that we have only one dust component composed of compact silicate grains of $0.1 \mu \mathrm{m}$ with an internal density of $3.6 \mathrm{~g} \mathrm{~cm}^{-3}$. No PAHs are included in this stage of the modeling. This modeling step yields the overall gas+dust density and temperature structure of the disk, under the assumption that the dust and the gas are fully mixed, i.e. that dust sedimentation has not yet taken place. These models also assume that the gas temperature equals the temperature of the dust, which is an assumption that may break down in the upper layers of the disk (Jonkheid et al. 2004; Kamp \& Dullemond 2004; Nomura \& Millar 2005). The disk models resulting from this procedure have a flaring shape with a slightly puffed-up inner rim, as described in DD04a. The rounding-off of the inner rim, as proposed and modeled by Isella \& Natta (2005) is not included in this model.

The second step starts from these hydrostatic disk structure models, but adds a component of PAHs to the dust (correcting the density of the $0.1 \mu \mathrm{m}$ silicate grains such that the total dust abundance remains 0.01 by mass). The PAHs are assumed to be of a single type and size: we take them to be neutral $\mathrm{C}_{50} \mathrm{H}_{20}$ and assume a cross-section, for friction with the gas, of $10^{-14} \mathrm{~cm}^{2}$ (the optical cross sections are discussed below). The assumption of this particular form of PAH is an arbitrary one, and the strength of the PAH features as well as the feature strength ratio may strongly depend on this choice. A detailed chemical calculation of the hydrogenation state and charge is the only way to remove some arbitrariness (Visser et al. 2007), but the size of the PAHs remains unknown. However, since this paper only intends to demonstrate the effect of sedimentation in disks with PAHs, a reasonable choice such as $\mathrm{C}_{50} \mathrm{H}_{20}$ seems justified.

The time-dependent sedimentation of both dust components is then modeled following the treatment of DD04b. The main difference with DD04b is the underlying disk structure model: in that paper a simple analytic model was used, while here we use the self-consistent hydrostatic model of DD04a (step 1).

The third step is to compute the SED of the disk at $10^{6}$ years after the start of the simulation. As in DD04b we apply a 2-D axisymmetric Monte Carlo radiative transfer code called RADMC (first used in Dullemond \& Dominik 2004a; see Appendix A for further information). For the $0.1 \mu \mathrm{m}$ silicate grains we assume the opacity of Laor \& Draine (1993) and we treat these grains as "thermal" grains. For the PAHs we use the opacities of Li \& Draine (2001, henceforth LD01), and treat the particles as quantum-heated grains. This quantum behavior is treated in a multi-photon "thermal continuous" approach (Guhathakurta \& Draine 1989; Siebenmorgen et al. 1992; Manske \& Henning 1998; Draine \& Li 2001). In this approach the vibrational states of individual PAHs are assumed, at each given moment, to be well described by a single temperature. However, over time, in the course of seconds to hours, this temperature fluctuates due to discrete heating events by stellar photons. The cooling in between the discrete heating events is assumed to be classical continuous thermal cooling (Guhathakurta \& Draine 1989). The "multi-photon" aspect of the method allows for the possibility that a PAH has not yet cooled down to its "base temperature" before a new discrete heating event (i.e. a photon absorption event) occurs. This method requires the solution of the temperature probability function $P(T)$. This function describes which fraction of the time the grain statistically has a given temperature. Once $P(T)$ is known, the emissivity per gram of PAH "dust" is: $\int_{0}^{\infty} P(T) B_{v}(T) \kappa_{v} \mathrm{~d} T$, where $\kappa_{v}$ is the opacity of the PAHs in $\mathrm{cm}^{2} /$ gram-of-PAH from LD01. Using the ray-tracing tools of the radiative transfer program the spectrum of the disk can then be computed.

Apart from exciting the PAHs to high temperatures, highenergy stellar photons can also destroy them (Allain et al. 1996). This is of particular concern close to the star where 


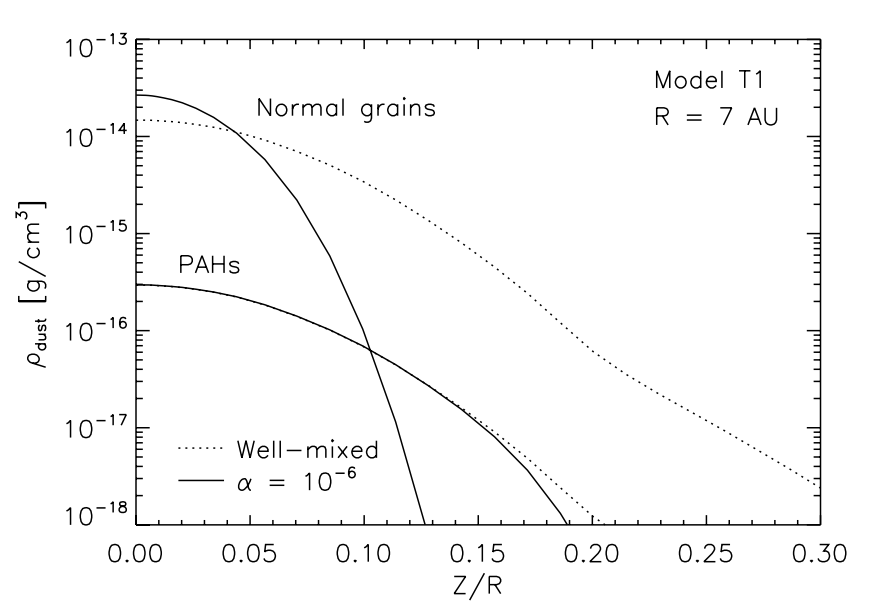

Fig. 1. The vertical distribution of dust and PAHs for the fully mixed case (dotted) and the case of turbulence at a level of $\alpha=10^{-6}$ (solid). These curves represent the vertical cross-section at $7 \mathrm{AU}$ in model T1. The fully mixed case (dotted) can be seen as the initial condition of our models, where the dust-to-gas ratio everywhere is the same as the assumed interstellar value (1:100).

multi-photon events (i.e. two absorption events within a time shorter than the cooling time) can boost the peak excitation of the grain to beyond its evaporation energy (Guhathakurta \& Draine 1989; Siebenmorgen et al. 1992). We assume the destruction energy/temperature to be $2000 \mathrm{~K}$ (H04). In H04 it was assumed that if a PAH molecule spends more than $10^{-8}$ of its life time in an excited state with a temperature above $2000 \mathrm{~K}$, then the PAH is removed, i.e. the PAH is removed if $\int_{2000 \mathrm{~K}}^{\infty} P(T) \mathrm{d} T>10^{-8}$. Here were adopt a slight variation of this. PAHs are mostly well mixed in vertical direction, even for relatively low turbulence (see e.g. Fig. 1). Thus PAHs usually spend only a short time in the disk's surface layers, while residing most of their life deep within the disk where they are shielded from destructive stellar radiation. While the PAH destruction can kill PAHs in the surface layers, new PAHs will be turbulently mixed to the surface. We therefore compute in our model the vertically averaged PAH destruction probability: if $p_{\mathrm{d}}(z)$ is the probability of a PAH being destroyed within the disk life time at height $z$ above the midplane, then the vertically averaged probability is given by $\left\langle p_{\mathrm{d}}\right\rangle=\int_{0}^{\infty} \rho_{\mathrm{PAH}}(z) p_{\mathrm{d}}(z) \mathrm{d} z / \Sigma_{\mathrm{PAH}}$, where $\rho_{\mathrm{PAH}}$ and $\Sigma_{\mathrm{PAH}}$ are the PAH volume density and surface density respectively. If this vertically averaged destruction probability exceeds $10^{-8}$, we remove the PAHs at each $z$ above the midplane. Otherwise we keep them at all $z$. We repeat this procedure for each radius $R$.

The basic elements of the method of calculating PAH emission have been described by Siebenmorgen et al. (1992). Earlier work on PAH modeling in protoplanetary disks was presented by Manske \& Henning (1999), H04, G06 and Visser et al. (2007).

\subsection{Scans of the parameter space}

We have produced a series of models, varying the stellar type, the strength of the turbulence and the abundance of PAHs. For the stellar parameters we take two cases: a T Tauri star with $M_{*}=0.5 M_{\odot}, R_{*}=2.5 R_{\odot}, T_{*}=4000 \mathrm{~K}$, and a Herbig star with $M_{*}=2.5 M_{\odot}, R_{*}=2.5 R_{\odot}, T_{*}=10000 \mathrm{~K}$. We use Kurucz models for these stellar spectra, and assume for our standard models that they have no UV excess due to accretion or other processes. This may not be a good assumption, in particular for $\mathrm{T}$ Tauri
Table 1. The parameters for the model-series. Each series represents 5 models with 5 different values of the turbulent $\alpha$-parameter: $\alpha=$ $10^{-2}, 10^{-3}, 10^{-4}, 10^{-5}, 10^{-6}$. Column 1: series name, Col. 2: stellar mass, Col. 3: stellar radius, Col. 4: stellar effective temperature, Col. 5: inner radius of the disk, Col. 6: PAH abundance by mass with respect to large grains. All disks have $M_{\text {disk }}=0.01 M_{\odot}, \Sigma \propto R^{-1}$ and $R_{\text {out }}=100 \mathrm{AU}$.

\begin{tabular}{c|ccc|cc}
\hline \hline Name & $M_{*} / M_{\odot}$ & $R_{*} / R_{\odot}$ & $T_{*}[\mathrm{~K}]$ & $R_{\text {in }} / \mathrm{AU}$ & Abun \\
\hline T1 & 0.5 & 2.5 & 4000 & 0.07 & 0.02 \\
T2 & 0.5 & 2.5 & 4000 & 0.07 & 0.005 \\
H1 & 2.5 & 2.5 & 10000 & 0.7 & 0.02 \\
H2 & 2.5 & 2.5 & 10000 & 0.7 & 0.005 \\
\hline
\end{tabular}

stars, but since much of the UV power is at wavelengths that are difficult to access observationally, these UV excesses are not easy to measure. As a lower limit we assume them to be absent, except in Sect. 3.7 where the effect of UV excess is explored.

For each of these stellar types we run models with 5 different values of the $\alpha$-parameter of turbulence: $\alpha=$ $10^{-2}, 10^{-3}, 10^{-4}, 10^{-5}, 10^{-6}$. Each of these models is run with two different PAH abundances: $\rho_{\mathrm{PAH}}^{0} / \rho_{\text {bigdust }}^{0}=0.005$ and $0.02^{1}$, where $\rho_{\mathrm{PAH}}^{0}$ and $\rho_{\text {bigdust }}^{0}$ are the initial mass density in PAHs and in big $(0.1 \mu \mathrm{m})$ grains respectively at the start of each simulation. This makes in total 20 models that we present here. Table 1 summarizes the models.

Except for the inner radius, the disk parameters are the same for all models. We take a disk mass of $M_{\text {disk }}=0.01 M_{\odot}$ for the gas+dust, assuming a dust-to-gas mass-ratio of 0.01 . The surface density profile is assumed to be a powerlaw: $\Sigma(R) \propto R^{-1}$. The disk has an outer edge of $R_{\text {out }}=100 \mathrm{AU}$.

\section{Results}

\subsection{Sedimentation}

The sedimentation of dust in disks in the context of the disk's appearance has been described in detail by DD04b. We show the resulting vertical dust distributions for the thermal grains and for the PAHs in Fig. 1. The normal grains sediment very deeply while the PAHs mostly are unaffected, except in the very upper layers.

\subsection{Feature strengths}

In Fig. 2 the SEDs of the models are shown for the PAH abundance of 0.02 relative to the full dust density. The models with $\alpha=10^{-4} \ldots 10^{-6}$ have PAH feature-over-continuum strengths that are stronger than typically observed, but a reduction of the $\mathrm{PAH}$ abundance can correct for this.

The results show that the PAH features are strongly affected by the sedimentation, and they are more prominent for weaker turbulence. Even for a relatively high level of turbulence $\left(\alpha=10^{-2}\right)$ the PAH features are enhanced by the sedimentation of the larger (thermal) grains. This has consequences for any analysis of observed spectra of PAHs in disks. As was shown in G06, to derive the PAH abundance from an observed disk spectrum without being too sensitive to the disk geometry one needs to analyze the feature-over-continuum ratios. If one does this using a model without sedimentation, one would need a higher PAH abundance to match the observed feature strengths than if

\footnotetext{
1 A PAH dust abundance of 0.02 amounts to about $1 \times 10^{-5} \mathrm{C}$-atoms in PAHs for each $\mathrm{H}$-atom in the disk, which is about one-third of the abundance taken by $\mathrm{H} 04$.
} 

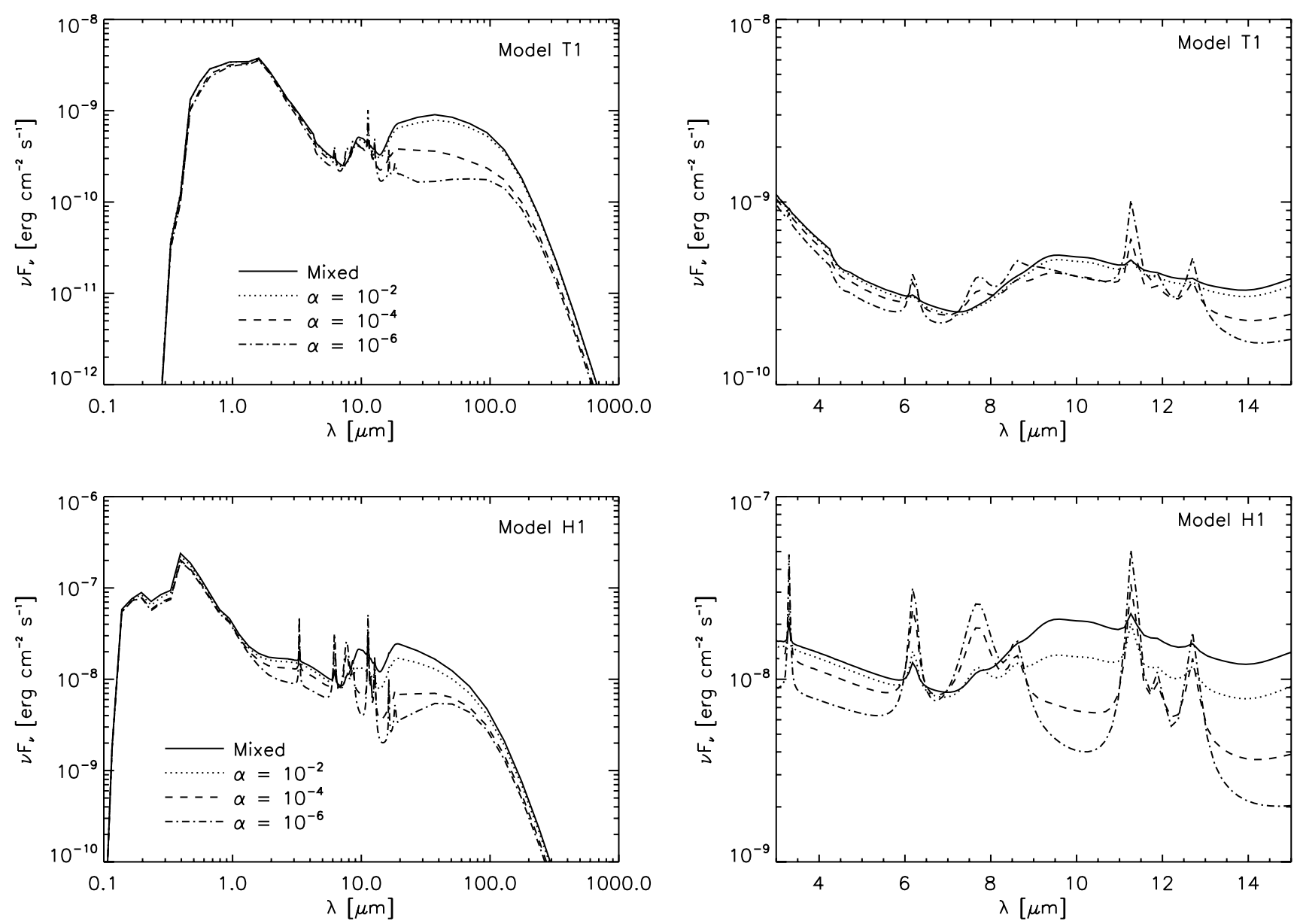

Fig. 2. The SEDs of models T1 (upper panels) and H1 (lower panels) of this paper, for an inclination of $45^{\circ}$, at $10^{6}$ years observed at a distance of 100 parsec. Left panels: the full SEDs, right panels: enlargement of the PAH-feature region. The curves are as follows. Solid: before sedimentation, all other curves after sedimentation $\left(t=10^{6}\right.$ years) for several values of $\alpha$ : dotted $\alpha=10^{-2}$, dashed $\alpha=10^{-4}$, dot-dashed $\alpha=10^{-6}$.

one includes the feature-enhancement effect caused by sedimentation of the "large" grains.

As can be seen in Fig. 2, there are two effects of enhancing the PAH features. The first is that the continuum drops strongly, and the second is that the absolute flux in the feature increases. Both effects can be understood by realizing that for low $\alpha$ the large $(0.1 \mu \mathrm{m})$ grains sediment well into the disk, while the PAHs hardly sediment at all. In the most extreme case the large grains therefore no longer absorb stellar light, reducing the continuum (which is for a significant fraction produced by these large grains), while the PAHs absorb all the stellar light, increasing the absolute PAH feature strength. The remainder of the continuum between the PAH features comes from two sources: according to LD01 the PAH opacity has a pseudo-continuum between the features. So even disks consisting purely of PAHs would have some level of emission between the features. But in this case, another strong contribution to the inter-feature continuum comes from the thermal emission from the disk midplane region (see e.g. Chiang \& Goldreich 1997). Note that our use of fully frequency-dependent multi-dimensional radiative transfer automatically takes care of all these sources of feature and continuum emission.

An important difference between the models $\mathrm{T} 1$ ( $\mathrm{T}$ Tauri star) and H1 (Herbig Ae star) is that the PAH feature-overcontinuum strengths seem to be stronger for the Herbig star model. This is because PAH particles are best excited by very short-wavelength radiation. A Herbig star has a stronger UV luminosity relative to its total luminosity than a T Tauri star, assuming that there is no UV emission originating from accretion onto the stellar surface. PAHs have a much larger absorption cross-section to UV and optical photons than to near-infrared photons $^{2}$. For the $0.1 \mu \mathrm{m}$ silicate grains such a difference is also present, but far less extreme than in the case of PAHs, at least for uncharged ones. For this reason, PAHs are much more sensitive to the effective temperature of the star than the thermal silicate grains, and hence the PAH-continuum ratio is stronger in the case of Herbig stars for the same assumed PAH abundance relative to the $0.1 \mu \mathrm{m}$ silicate dust.

\subsection{SED shape}

The left panels of Fig. 2 show that not only are the features affected by the sedimentation, but also the overall SED shape. This effect is most clearly visible in the spectral region between 20 and $100 \mu \mathrm{m}$. For low turbulence, this region of the SED is suppressed: the total energy contained in this $20-100 \mu \mathrm{m}$ region of the SED is reduced for lower $\alpha$. This is a reflection of the fact that

2 Note, however, that Mattioda et al. (2005b) show that charged PAHs have a much stronger NIR opacity than the Li \& Draine opacities. For neutral PAHs this enhanced NIR opacity is not present. 
the $\tau_{*}=1$ surface of the disk (i.e. the surface where the irradiating stellar radiation is absorbed) is lowered toward the midplane when the dust sediments to the midplane. Thus the geometry of the disk becomes thinner. A geometrically thinner disk captures less radiation from the star and hence emits less radiation in the infrared. This effect was demonstrated by Miyake \& Nakagawa (1995) and Dullemond \& Dominik (2004b) who have shown that sedimentation can lead to the supression of the far-IR emission.

However, while the large grains sediment to the midplane, the PAHs stay high up. In principle they alone might be able to keep the disk flaring and keep the far-infrared flux strong. There are two reasons why this is not the case. First, in particular in the case of the T Tauri stars, the PAHs initially account for only a small fraction of the absorbed stellar radiation. The removal by sedimentation of the large grains from the surface layer removes a significant fraction of this local optical opacity, thereby causing the $\tau_{*}=1$ layer to move closer to the midplane, and hence suppress the mid- to far-infrared flux of the disk. Secondly, the PAHs have a very low opacity in the far-infrared. Typically this means that these grains will emit their energy at much shorter wavelengths, and can therefore not prevent the mid- to far-infrared flux of the disk from being suppressed by the sedimentation of the large grains.

The suppression of the mid- to far-infrared spectral region happens for low turbulence. In particular for the T Tauri star model the SED shape difference between $\alpha=10^{-2}$ and $\alpha=10^{-3}$ is negligible; only when going to $\alpha=10^{-4}$ does the SED shape suddenly change. For the Herbig star case this is less extreme, but still the SED shape difference between $\alpha=10^{-2}$ and $\alpha=10^{-3}$ is only minor, while the feature enhancement between these two models is strong. Going to even lower $\alpha$ the PAH features are enhanced even further, but the SED also starts to be strongly affected.

\subsection{Feature indicators as a function of $\alpha$}

To quantify the strength of the features, we define unbiased feature strength indicators. These strengths are measured by fitting a power law "continuum" between two points on both sides of the feature and computing the value $C$ of this "continuum" at the location of the feature peak. The feature-to-continuum strength indicator is then:

$\varphi=\frac{F-C}{C}=\frac{F}{C}-1$

where $F$ is the flux at the peak of the feature. For the abovementioned features the adjacent wavelengths we used are: 3.0 and $3.6 \mu \mathrm{m}$ for the $3.3 \mu \mathrm{m}$ feature, 6.7 and $8.3 \mu \mathrm{m}$ for the $7.7 \mu \mathrm{m}$ feature, 10.8 and $11.7 \mu \mathrm{m}$ for the $11.3 \mu \mathrm{m}$ feature, and 12.4 and $13.0 \mu \mathrm{m}$ for the $12.7 \mu \mathrm{m}$ feature. These feature strength indicators produce positive numbers for emission features and negative numbers for absorption features. Since the continuum is generally curved, the absence of a feature can lead to non-zero or even negative strength values. The more sophisticated feature indicators introduced by G06, with spline fits to the continuum and integrals over the feature profile, are less prone to such artifacts. However, for simplicity we prefer to employ the simpler power-law based feature strength indicators introduced above. However, to determine the true peak flux of the feature, the observation must have the right spectral resolution to truly resolve the profile of the feature. If we take the opacities of LD01 at face value then one needs a resolution of at least $R=100$ for the 11.3 feature, for instance. From the Spitzer observations of
G06 PAH feature profiles are found from which a similar constraint can be derived. Our resulting $F$ values also depend on our assumed PAH opacity table (LD01). Some caution is therefore needed, and the normalization of $F$ values (in our paper and in observations) should not be taken too literally. The trends we predict are more robust.

Another feature to quantify is the shape of the SED. In principle this involves many "shape-factors", but the most conspicuous shape modification by the sedimentation is the strength of the mid- to far-infrared as compared to the near infrared. We introduce two "slope values", $s_{30}$ and $s_{70}$, which are defined as the slopes $s$ of the power law line $v F_{v} \propto v^{s}$ that connects the measured fluxes at $5 \mu \mathrm{m}$ and either $30 \mu \mathrm{m}$ (for $s_{30}$ ) or $70 \mu \mathrm{m}$ (for $\left.s_{70}\right)$.

In Fig. 3 the PAH feature indicators and the SED slope indicators are shown for models T1, T2, H1 and H2 as a function of the strength of the turbulence. It is seen that all strength indicators have a stronger dependence on $\alpha$ for the T Tauri star models (T1 and T2) than for the Herbig star models (H1 and H2). Moreover, the difference in PAH feature strength between the models $\mathrm{H} 1$ and $\mathrm{H} 2$ and between $\mathrm{T} 1$ and $\mathrm{T} 2$ (i.e. a factor of 4 in vertically averaged PAH abundance) is less pronounced for the low-alpha cases than for the fully mixed cases. This is because once the large grains have sedimented very deeply, the PAHs dominate the photosphere anyway, even when the vertically averaged (i.e. initial) PAH abundance is reduced by a factor of 4 .

Even for $\alpha=10^{-2}$, which can be considered as reasonably strong turbulence, the features are enhanced compared to the fully mixed case. In particular for the Herbig star models the dependence of the enhancement on $\alpha$ is strongest at the higher $\alpha$ values: once the turbulence becomes very weak, further reduction of the turbulence is less effective. This is also related to the fact that once the PAHs dominate the photosphere, there is not much more enhancement that can be achieved by further reduction of the turbulence.

\subsection{Ratios of strength indicators: analysis of charge and hydrogenation state}

PAH molecules can exist as various types: they can have different sizes, shapes, hydrogenation states (i.e. the number of $\mathrm{H}-$ atoms at the edges) and charges. Each of these types of PAH also has different strengths of the PAH features. If the spectrum of the exciting radiation is known, one can compute the predicted ratios of feature strengths $(F-C)$ for a particular type of PAH and compare it to the observations. In this way one can estimate the dominant type of PAH present in that particular source, and this can then be compared to chemical models (Visser et al. 2007). This method has been used before to estimate the charge and hydrogenation state of PAHs in PDRs (Verstraete et al. 1996; Henning \& Klein 1997). As an illustration we show in Fig. 4 the spectra of model T1 at $\alpha=10^{-6}$ for three different PAH states.

For disk sources there are, however, a number of problems with this method. Firstly, multi-photon excitations cause these feature strength ratios to depend on the distance of the PAH molecule to the star, or the depth of the PAH molecule into the disk. The dependence of these ratios on radius vanishes when the PAH molecule is far enough away from the exciting source that multi-photon excitations do not play a role. For disks, multiphoton excitations do play a role, in particular for the $3.3 \mu \mathrm{m}$ feature, and these ratios are modified in a way that requires detailed modeling.

Another complexity arises due to the fact that PAHs that reside deeper in the disk, but not deep enough that they see no 

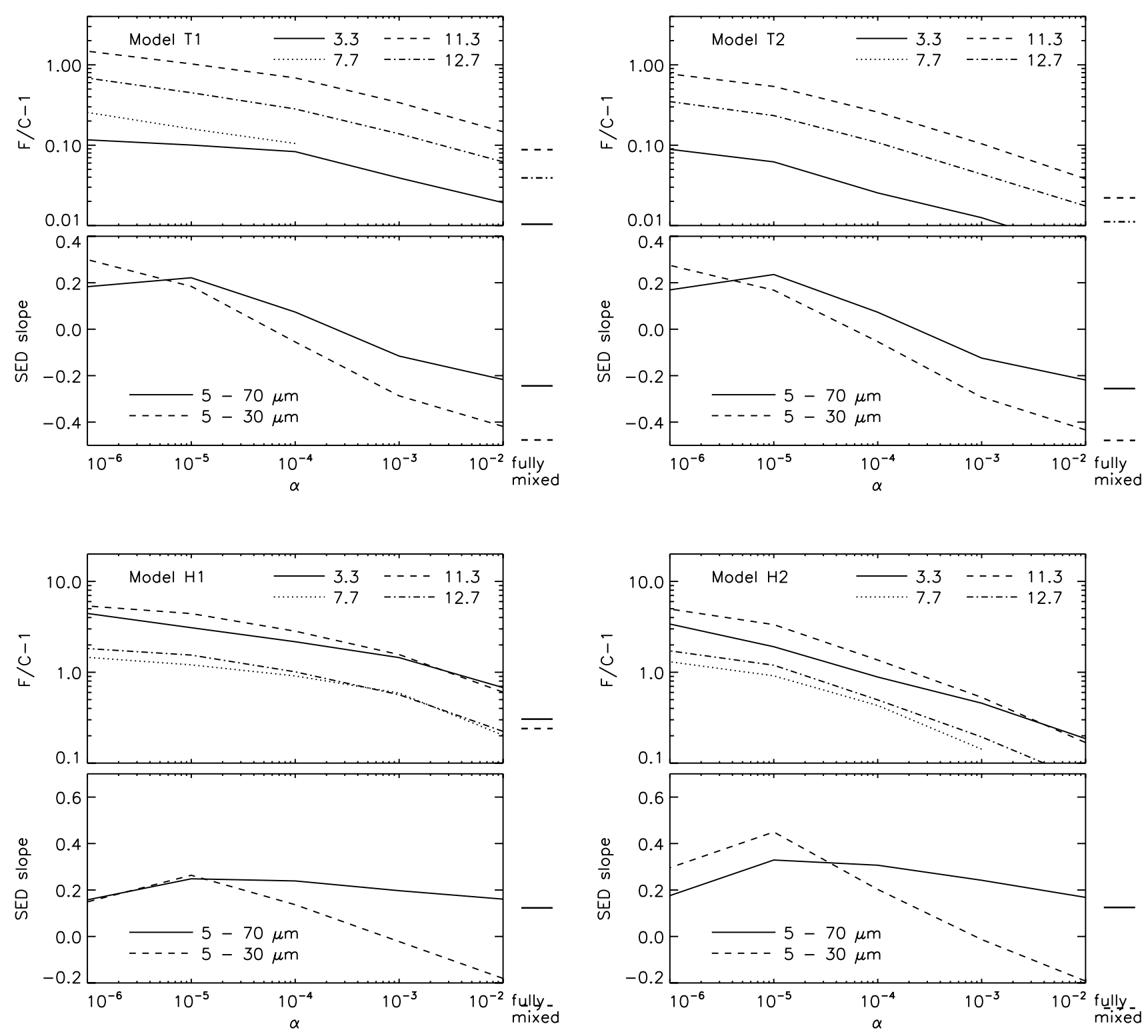

Fig. 3. The feature-to-continuum ratios and SED slopes as a function of the turbulence parameter $\alpha$ for both stellar types and both PAH abundances. Upper panels: the T Tauri star, lower panels: the Herbig star. Left panels: high PAH abundance (0.02 by mass), right panels: low PAH abundance (0.005 by mass). The short horizontal lines to the right of the plots are the values for fully mixed models. Note that the vertical scale for the T Tauri star case (upper panels) is different from that of the Herbig star case (lower panels).

stellar radiation at all, are excited by stellar radiation that has been reddened by the dust closer to the star, i.e. has a different spectral shape than direct starlight. The emission spectrum of these PAHs is therefore different from that from PAHs in the very upper layers of the disk.

Sedimentation of the larger grains changes all these conditions, and it is interesting to investigate to what extent these feature strength ratios are affected by the sedimentation. If they are strongly affected, then the measurement of feature strength ratios may not be easy to interpret. If not, then there is more hope that feature-strength ratios may directly provide clues to the dominant type of PAH particles.

For the ratios of the feature-to-continuum indicators the results can already be inferred from Fig. 3. Compared to the 11.3 and $12.7 \mu \mathrm{m}$ features, the $7.7 \mu \mathrm{m}$ feature strength is much stronger for the Herbig star models ( $\mathrm{H} 1$ and $\mathrm{H} 2$ ) than for the
T Tauri star models ( $\mathrm{T} 1$ and T2). This is even more the case for the $3.3 \mu \mathrm{m}$ feature: for the $\mathrm{T}$ models it is among the weakest features while for the $\mathrm{H}$ models it is among the strongest features. This is because the Herbig stars have sufficient UV photons that excite the $3.3 \mu \mathrm{m}$ feature directly or with only one additional photon. The $\mathrm{T}$ Tauri star spectrum is too cool for direct excitation of the $3.3 \mu \mathrm{m}$ feature, while this is less of a problem for the longer-wavelength features like the 11.3 and $12.7 \mu \mathrm{m}$ features. However, if proper chemistry of the PAHs would be included, the strong UV field of the Herbig stars might ionize the PAHs more, which would reduce the $3.3 \mu \mathrm{m}$ feature again. Including this kind of chemistry is the topic of another paper (Visser et al. 2007). Here we keep the state of the PAHs fixed.

For each star individually, the ratios of the feature-tocontinuum strengths as a function of $\alpha$ are reasonably stable. For the T1 model there is a slight change in the 3.3/11.3 ratio 


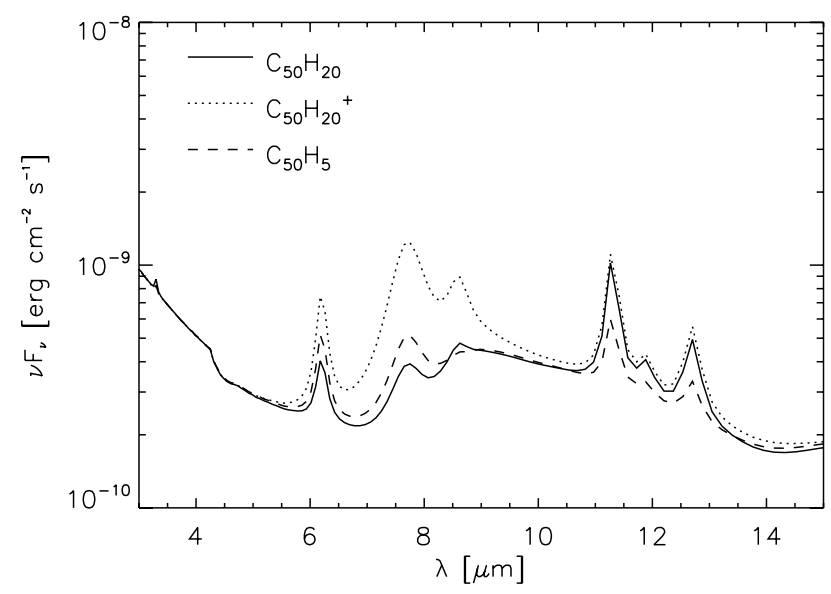

Fig. 4. The spectrum of model $\mathrm{T} 1$ for $\alpha=10^{-6}$, with three different types of PAH: neutral $\mathrm{C}_{50} \mathrm{H}_{20}, \mathrm{C}_{50} \mathrm{H}_{20}{ }^{+}$, and neutral $\mathrm{C}_{50} \mathrm{H}_{5}$. For the first and the third we take the opacity of Li \& Draine (2001). For the second (the charged one) we take the Li \& Draine opacity adapted by Mattioda et al. (2005a) for the increased near-infrared opacity.

with $\alpha$, changing by less than a factor of two between $\alpha=10^{-6}$ and $\alpha=10^{-2}$. The $7.7 \mu \mathrm{m}$ feature becomes unmeasureable for $\alpha>10^{-4}$. It artificially becomes negative as a result of the curvature in the SED at that point due to the onset of the $10 \mu \mathrm{m}$ silicate feature.

\subsection{The effect of the presence of VSGs}

So far we have assumed two grain sizes: the PAH molecules on the one hand and the "large" thermal dust grains on the other hand. In practice there will be a size distribution of particles, sedimenting down to different heights above the midplane. Or, alternatively, there might be so-called "Very Small Grains" (VSGs, Boulanger et al. 1988) in the disk. These grains are larger than PAHs but still small enough that they have a quantum-fluctuating thermal behavior. Also, by being still significantly smaller than the "thermal' grains of our model, they sediment only weakly, though slightly more than PAHs. Together with the PAHs, these VSGs will then dominate the disk's photosphere, and since VSGs have a different opacity $\kappa_{v}$ than PAHs, this may influence the results. In the extreme case that these VSGs are nonhydrogenated carbon grains, the opacity of these grains will be largely featureless. However, analysis of soot created in the laboratory, in which PAHs have been removed by elution, still show some $\mathrm{C}$-H out-of-plane bending mode features, in particular the $11.3 \mu \mathrm{m}$ feature, indicating that these soot particles are hydrogenated to a certain level (Jäger et al. 2006). Since these bands overlap with the PAH bands, such hydrogenated VSGs will have a similar behavior as the PAHs themselves. To maximize any possible effect of VSGs on the present model, we use pure carbon grains for our VSGs, i.e. grains without PAH-like features. The optical constants are taken from Preibisch et al. (1993).

We perform the test for the T Tauri star model with $\alpha=10^{-6}$. We introduce purely carbonaceous VSGs of $10^{5} \mathrm{C}$ atoms to the disk with the same initial mass-abundance as the PAHs. We treat their heating and cooling in the same quantum way as the PAHs.

The results are shown in Fig. 5. Due to the fact that VSGs do not sediment very deeply into the disk, they are also well in sight of the stellar radiation, and compete with the PAHs for exciting stellar photons. Therefore the PAH emission is slightly reduced and (featureless) VSG emission is introduced. This fills in the continuum between the PAH features, thereby weakening them, as seen in Fig. 5.

Interestingly, the far-infrared emission level is hardly affected. Even with VSGs which settle slightly, but still much less than the normal "thermal" grains, the far-infrared emission is strongly reduced compared to the case with $\alpha=10^{-2}$. The reason is, firstly, that the emission from these VSGs is peaked at shorter wavelengths than the normal thermal grain emission. This can be seen in Fig. 5 where the dashed line has a bump around $20-30 \mu \mathrm{m}$. The second reason is that the VSGs still represent only a small fraction of the dust. Most of the dust, being in the form of larger grains, sediments down and the optical depth of the remaining VSGs in the surface layers is not enough to prevent the mid- to far-infrared flux from being reduced.

\subsection{UV excess due to accretion}

Varying the $\alpha$ parameter of turbulence also is believed to affect the accretion rate in the disk, and hence affect the amount of UV excess produced by the magnetospheric accretion column on the surface of the star. For typical T Tauri accretion rates of about $\dot{M}=10^{-8} M_{\odot} /$ yr the total luminosity of this UV excess is rather small compared to the stellar luminosity. The luminosity can be estimated using the following formula (Calvet et al. 1998, henceforth CG98):

$L_{\text {column }}=\frac{G M_{*} \dot{M}}{R_{*}}\left(1-\frac{R_{*}}{R_{\text {inf }}}\right)$

where $R_{\text {inf }}$ is the inner radius of the gas disk, where the magnetospheric infall starts. Using $R_{\mathrm{inf}}=5 R_{*}$ (CG98) and using our values for the T Tauri star model we obtain $L_{\text {column }}=0.05 L_{\odot}$ for an accretion rate of $\dot{M}=10^{-8} M_{\odot} / \mathrm{yr}$, which is modest compared to the stellar luminosity.

For the disk model T1 that we have we can estimate an accretion rate of roughly $\dot{M}=8 \times 10^{-9} M_{\odot} / \mathrm{yr}$ for $\alpha=0.01$. This is based on standard formulae for viscous disks: $\dot{M}(R)=$ $2 \pi R^{2} \Sigma\left|v_{R}\right|$ with $v_{R}=-(3 / 2) v / R$. Here the viscosity $v$ is given by $v=\alpha c_{\mathrm{S}}^{2} / \Omega_{\mathrm{K}}$ with the isothermal sound speed squared given by $c_{\mathrm{s}}^{2}=k T /\left(2.3 m_{\mathrm{p}}\right)$, and $\Omega_{\mathrm{K}}=\sqrt{G M_{*} / R^{3}}$. In our T1 model at $R=1 \mathrm{AU}$ we have $\Sigma=162 \mathrm{~g} / \mathrm{cm}^{2}$ (giving with $\Sigma \propto 1 / R$, $R_{\text {out }}=100 \mathrm{AU}$ and $R_{\text {in }}=0.07 \mathrm{AU}$ a disk mass of $0.01 M_{\odot}$ ) and the temperature $T$ is estimated to be $140 \mathrm{~K}$ at that location. This temperature estimate is based on a simple flaring angle argument similar to Chiang \& Goldreich (1997). We can now scale $\dot{M}$ as the turbulence parameter $\alpha$ is varied. So now the variation of $\alpha$ has two effects: it affects the sedimentation and the UV excess.

We assume that the accreting material falls onto the star in very localized spots. The total covering fraction of the spots on the stellar surface is assumed to be $C=0.003$ (in accordance with estimates of CG98 for T Tauri stars). We now make the simple assumption that the emission from these hot spots on the stellar surface are purely thermal blackbody. This is a reasonable approximation, as can be seen in Fig. 3 of CG98. The effective temperature of these hot spots (accretion columns) is then

$T_{\text {col }}=\left(\frac{L_{\text {column }}}{4 \pi C R_{*}^{2} \sigma}\right)^{1 / 4}$,

where $\sigma$ is the Stefan-Boltzmann constant. Assuming that this is blackbody emission we now replace the usual stellar luminosity $L_{*, v}$ in the models of this paper by,

$L_{\mathrm{center}, v}=L_{*, v}+4 \pi C R_{*}^{2} \pi B_{v}\left(T_{\mathrm{col}}\right)$. 

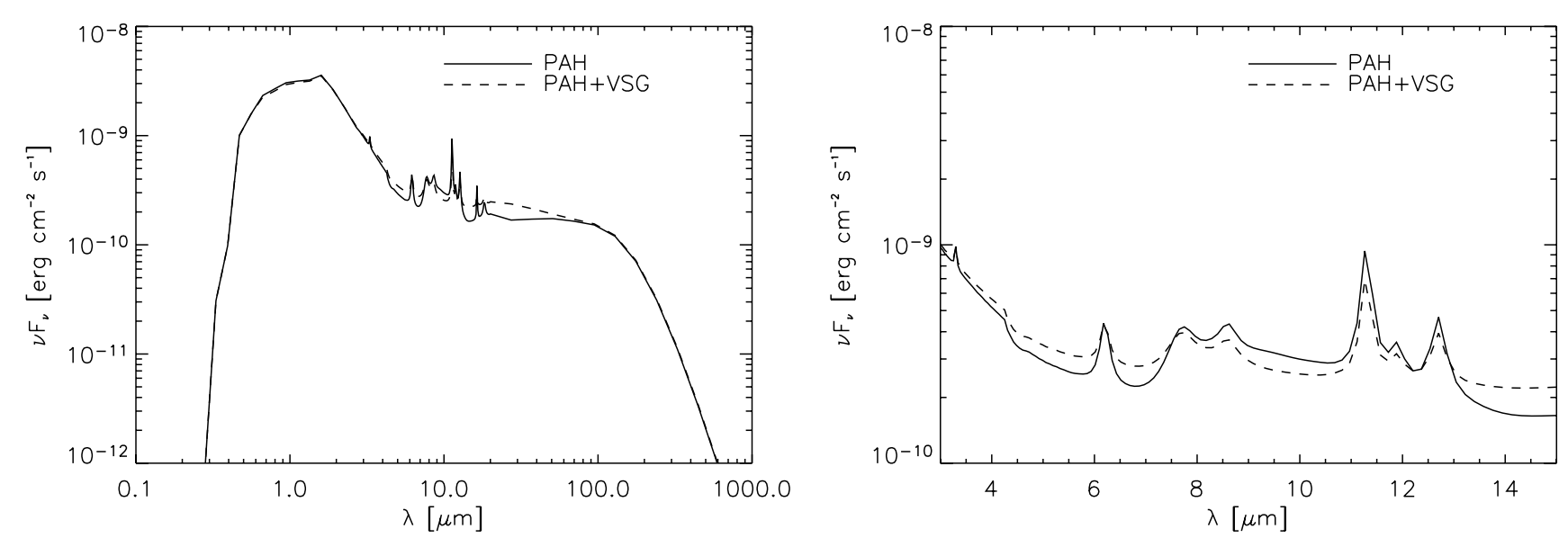

Fig. 5. Comparison between a model without VSGs (solid line) and with VSGs at an equal abundance as the PAHs (dashed line). Left: full SED. Right: enlargement. The model shown is the T Tauri star model with $\alpha=10^{-6}$.

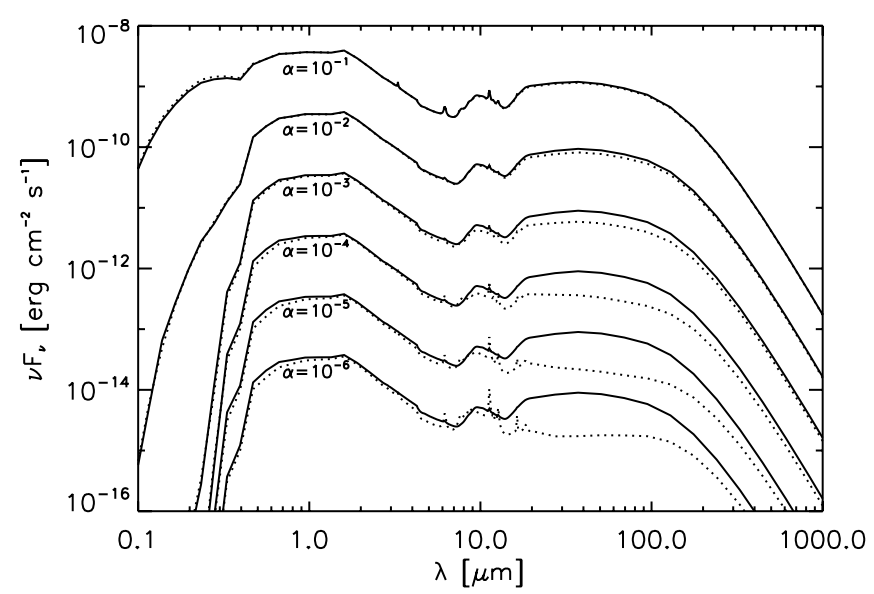

Fig. 6. The results of the models of Sect. 3.7 in which the UV excess due to magnetospheric accretion is included, and assumed to vary with $\alpha$ because $\alpha$ also controls the accretion rate. The spectra are shifted downward to prevent overlap. The top spectrum is the unshifted one and shifts were done in powers of 10 downward. Solid lines are the SEDs before sedimentation, dotted lines after sedimentation.

Using this new central luminosity (which now varies with $\alpha$ ) we can now rerun the various models of model set T1 again. Since now also the initial spectrum before sedimentation may vary (because the central luminosity source varies with $\alpha$ ) we plot for each value of $\alpha$ both the spectrum before and the spectrum after sedimentation. The results are shown in Fig. 6.

We see from this Figure that the results are hardly affected by the UV excess for $\alpha \lesssim 10^{-2}$ (compared to the results for the T1 model without UV excess shown in Fig. 2). This is because the total energy in the UV excess for this value is so low that it does not really affect the PAH strength. This is true in spite of the fact that a UV photon is more effective in exciting PAHs than an optical photon from the star. For $\alpha=10^{-1}$ we see that the PAH features are boosted due to the strong UV excess, but at that value of $\alpha$ the dust is well-mixed everywhere and the effect on the PAH emission due to sedimentation of the larger grains does not occur here.

\section{Discussion}

\subsection{PAH feature strength: other factors}

The strength of the PAH features for any given value of the turbulent mixing parameter $\alpha$ depends not only on the strength of the turbulence, but on a number of other factors as well. The most important factor is the abundance of PAHs. This is a large unknown, making it difficult to separate effects of sedimentation and abundance. A second important factor is the size of the "large" thermal grains. We have taken them all to be $0.1 \mu \mathrm{m}$ in size. If there is a size distribution toward smaller grain sizes, then the effects described in this paper may be somewhat suppressed. The model with the inclusion of VSGs (Sect. 3.6) shows a similar situation. However, many $10 \mu \mathrm{m}$ spectra of disks show that the minimum grain sizes are larger than $1 \mu \mathrm{m}$ (e.g. van Boekel et al. 2005). In that case the effects may be enhanced.

Another effect that can enhance the PAH features, as discussed by Geers et al. (2006), is the presence of a large inner hole in the disk, such as those detected around TW Hydra (Calvet et al. 2002) or CoKu Tau-4 (D'Alessio et al. 2005). The inner hole causes the thermal dust continuum emission in the nearinfrared to be suppressed, so that the PAH lines (which originate at much larger radii) stand out in stronger contrast. Such an effect was found observationally by Brown et al. (submitted to ApJ).

\subsection{Correlation between SED shape and PAH feature strength}

As shown above, while lowering the turbulence can enhance the PAH features, it also tends to change the overall SED shape: the disk becomes geometrically thin enough that the mid- to farinfrared continuum flux of the disk drops. Such SEDs with suppressed mid- to far-infrared SED are indeed observed. Meeus et al. (2001) found that Herbig stars can be classified into two groups: those with strong far-infrared flux ("group I") and those with weak far-infrared flux ("group II"). They suggested that the group I sources have disks with strongly flaring geometry (they are "geometrically thick") while group II sources have disks with non-flaring geometry. Dullemond (2002) and Dullemond $\&$ Dominik (2004a) have presented 2-D disk structure models in which it was shown that such geometries can indeed exist and can explain these two groups of sources. In the latter paper it was suggested that grain growth by coagulation might convert 
a flaring group I disk into a flatter, or even "self-shadowed" group II disk: the growth of grains lowers the overall optical depth of the disk, which suppresses the flaring. But sedimentation could also have this effect: if the grains sediment, they drag the disk photosphere down with them, because the dust is the primary carrier of opacity (Chiang et al. 2001; Dullemond \& Dominik 2004b). This is the effect seen in the simulations presented here.

If the distinction between group I and group II sources is indeed purely an effect of sedimentation, then the calculations of this paper make a prediction regarding the correlation between the strength of the PAH features and the shape of the SED: group II sources should have stronger PAH features than group I sources. However, observations seem to show the opposite trend: group II sources typically tend to have weaker PAH features, at least for Herbig Ae/Be stars (Acke et al. 2004). For T Tauri stars the statistics are not good enough to make strong conclusions: G06 find only 3 firm detections of PAH emission among their $38 \mathrm{~T}$ Tauri stars. Bouwman et al. (in prep.), in a small sample of 7 sources, see a tentative trend similar to Acke et al. (2004), but the statistics are very rough. However, for the Herbig stars the trend is clear. Radiative transfer models in which the flatter disk geometry of group II sources is attributed to a geometric flattening of both the large grain component and the PAH-component do correctly show the observed trend (G06). Therefore we conclude that, if the existence of group II sources is due to a flattening of the disk, then this can not be the result of dust sedimentation alone (as suggested by Dullemond \& Dominik 2004b and by Chiang et al. 2001). If it were, then an opposite trend of PAH strength with SED shape would be seen. It therefore must be a combination of effects, presumably dominated by grain growth (see below). If a large portion of VSGs are present, the predicted opposite trend is weakened. Still, the trend cannot be reversed: it is either opposite to observations of Herbig stars or absent.

\subsection{Grain growth: effect on feature strength and SED shape}

Instead of relying on the vertical downward sedimentation motion of particles to make the disk thinner (which works only for the thermal grains, and therefore enhances the PAH features), we may invoke the removal of a significant fraction of both PAHs and thermal grains through aggregation (for a review of aggregation, see Dominik et al. 2007). However, for PAHs this process is still largely unexplored. Moreover, if we would apply the current knowledge of aggregation of thermal grains to both thermal grains and PAHs, then this would remove these grains so effectively and rapidly that it is inconsistent with the large number of very optically thick flaring disks observed (see e.g. Dullemond $\&$ Dominik 2005). For these reasons we will not make quantitive models of this process here. It is, however, one possible explanation of why the trend of PAH feature strength observed among Herbig stars is opposite to what is found here. The enhancement of PAH features by sedimentation will still be important. However, if aggregation is the dominant factor in the flattening of the disk, then the PAHs are more strongly removed than the larger grains because according to aggregation theory the smallest particles aggregate the fastest.

\section{Conclusion}

This paper shows that sedimentation can enhance the infrared spectral signatures of small grains in a protoplanetary disk, in particular of PAHs. This phenomenon already occurs for turbulence levels as high as $\alpha=10^{-2}$, but becomes stronger for lower values of $\alpha$. Inferring PAH abundances from observed spectra using fully mixed models will presumably over-estimate the vertically-averaged PAH abundance: with sedimentation the same PAH feature-over-continuum strengths can be achieved for much lower vertically-averaged PAH abundances. A similar effect will take place for any other small grains compared to the larger grain population. For low turbulence the grain size distribution inferred in the surface layers of a disk may therefore not be indicative of the vertically averaged grain size distribution: the larger grains may have sedimented out of sight. How this quantitatively affects the analysis of (non-PAH) grain sizes in disks using infrared spectroscopy must be investigated in another paper.

In addition to enhancing the PAHs, also the overall SED shape is changed by lowering the $\alpha$. The model predicts that, if sources with low mid- to far-infrared flux ("group II" in the classification of Meeus et al. 2001) originate through sedimentation of dust, they should have stronger PAH features than sources with strong mid- to far-infrared flux ("group I"). Observations show the opposite trend, at least for Herbig stars (Acke \& van den Ancker 2004), showing that the differences between group I and group II sources cannot be attributed to a different level of turbulence. We speculate that grain growth (in addition to sedimentation) may produce a trend that is more consistent with the observations, but at present it is not clear how to quantitatively model this for the PAHs.

Acknowledgements. We thank Carsten Dominik for useful comments. Astrochemistry in Leiden is supported by a NWO Spinoza grant and a NOVA grant, and by the European Research Training Network "The Origin of Planetary Systems" (PLANETS, contract number HPRN-CT-2002-00308).

\section{Appendix A: Monte Carlo radiative transfer with PAHs}

The radiative transfer code we use for this paper, RADMC (first used in Dullemond \& Dominik 2004a) is a Monte Carlo code based on methods that are well described in the literature. The basic method is that of Bjorkman \& Wood (2001), which is a highly reliable Monte Carlo method ensuring flux conservation and without iteration. The RADMC code includes various modifications of this method: it uses a variable spacing in frequency space, it treats absorption as a continuous process when it comes to the effect the package has on the temperature of the cell, but it treats absorption as a discrete process in the directional change of a photon package (see Lucy 1999). When all photon packages have been launched and have left the system, the temperature of the dust is known everywhere in the disk. Using a ray-tracing program (for which we use a code called RADICAL) we can then compute images and spectra.

Including PAHs in the code is a non-trivial matter. As has been described in the main text, the quantum heated PAH "grains" do not have an equilibrium temperature, but have instead vibrational modes that can be excited and will quickly radiate away their energy before a new UV photon comes along to re-excite these vibrational levels. In most cases, if the PAH molecule is not too small, then at any given time the vibrationally excited state of the molecule can be reasonably well described with a temperature $T$ (Draine \& Li 2001). This temperature will, however, fluctuate in time. The RADMC code, being based on the Bjorkman \& Wood algorithm, assumes that the dust has a clearly defined (non-fluctuating) temperature. We do not rule out that it might be possible to reformulate the Bjorkman \& Wood algorithm to accomodate complete temperature distribution functions $P(T)$, but we estimate that, if 
it is possible, it will slow down the code considerably. Instead we make use of the property of PAHs that they are predominantly excited by UV and optical photons, which almost exclusively originate from the stellar surface and its possible accretion column. Thermal emission from the disk may excite the PAHs slightly, but this is always of much less importance than the radiation from the central stellar source. Therefore we have implemented a three-stage procedure to implement PAH emission in the Monte Carlo computation.

1. First run RADMC without PAH emission, but with PAH absorption. Any photon package that hits a PAH molecule will be removed from the simulation. But all the energy that the photon package has stored in the PAH population in each cell is memorized, and at the end of the first RADMC run this is stored to a file.

2. Then a separate code called PAHCODE is run. This reads in the file dumped by RADMC, which tells how much energy of primary stellar photons is pumped into the PAH population in each cell, and the temperature distribution function $P(T, r, z)$ is then calculated. From this, the emissivity $\epsilon_{v}(r, z)$ of the $\mathrm{PAH}$ molecules is calculated at each point in the disk and for each frequency.

3. The second run of RADMC absorbs and removes photons hitting PAH molecules as in step 1, but at the same time it will also emit new photon packages from the PAH population, with a frequency distribution according to the shape of the emissivity $\epsilon_{v}$. In this way energy is again conserved, although not as strictly as in the original Bjorkman \& Wood method. The second run is - at least in principle - necessary, because the thermal dust may absorb photons that originate from the PAHs, and therefore the PAH emission must be included in this second RADMC run.

Once these three steps have been performed, the temperatures of the thermal dust grains are computed and the temperature distribution function of the PAHs (and the corresponding emissivity function) is also known. Now the ray-tracing routines from the code RADICAL will read this information in, and produce spectra and images.

\section{References}

Abergel, A., Bernard, J. P., Boulanger, F., et al. 1996, A\&A, 315, L329 Acke, B., \& van den Ancker, M. E. 2004, A\&A, 426, 151
Allain, T., Leach, S., \& Sedlmayr, E. 1996, A\&A, 305, 602 Bjorkman, J. E., \& Wood, K. 2001, ApJ, 554, 615 Boulanger, F., Beichman, C., Desert, F. X., et al. 1988, ApJ, 332, 328 Calvet, N., \& Gullbring, E. 1998, ApJ, 509, 802

Calvet, N., D'Alessio, P., Hartmann, L., et al. 2002, ApJ, 568, 1008

Chiang, E. I., \& Goldreich, P. 1997, ApJ, 490, 368

Chiang, E. I., Joung, M. K., Creech-Eakman, M. J., et al. 2001, ApJ, 547, 1077 Churchwell, E., Whitney, B. A., Babler, B. L., et al. 2004, ApJS, 154, 322

D’Alessio, P., Hartmann, L., Calvet, N., et al. 2005, ApJ, 621, 461

Dominik, C., Blum, J., Cuzzi, J. N., \& Wurm, G. 2007, in Protostars and Planets V, ed. B. Reipurth, D. Jewitt, \& K. Keil, 783

Draine, B. T., \& Li, A. 2001, ApJ, 551, 807

Dubrulle, B., Morfill, G., \& Sterzik, M. 1995, Icarus, 114, 237

Duchêne, G., Ménard, F., Stapelfeldt, K., \& Duvert, G. 2003, A\&A, 400, 559

Dullemond, C. P. 2002, A\&A, 395, 853

Dullemond, C. P., \& Dominik, C. 2004a, A\&A, 417, 159

Dullemond, C. P., \& Dominik, C. 2004b, A\&A, 421, 1075

Dullemond, C. P., \& Dominik, C. 2005, A\&A, 434, 971

Furlan, E., Hartmann, L., Calvet, N., et al. 2006, ApJS, 165, 568

Geers, V. C., Augereau, J.-C., Pontoppidan, K. M., et al. 2006, A\&A, 459, 545

Guhathakurta, P., \& Draine, B. T. 1989, ApJ, 345, 230

Habart, E., Natta, A., \& Krügel, E. 2004, A\&A, 427, 179

Habart, E., Natta, A., Testi, L., \& Carbillet, M. 2006, A\&A, 449, 1067

Henning, T., \& Klein, R. 1997, A\&SS, 255, 53

Isella, A., \& Natta, A. 2005, A\&A, 438, 899

Jäger, C., Krasnokutski, S., Staicu, A., et al. 2006, ApJS, 166, 557

Jonkheid, B., Faas, F. G. A., van Zadelhoff, G.-J., \& van Dishoeck, E. F. 2004, A\&A, 428, 511

Kamp, I., \& Dullemond, C. P. 2004, ApJ, 615, 991

Kenyon, S. J., \& Hartmann, L. 1987, ApJ, 323, 714

Lagage, P.-O., Doucet, C., Pantin, E., et al. 2006, Science, in press

Laor, A., \& Draine, B. T. 1993, ApJ, 402, 441

Li, A., \& Draine, B. T. 2001, ApJ, 554, 778

Lucy, L. B. 1999, A\&A, 344, 282

Manske, V., \& Henning, T. 1998, A\&A, 337, 85

Manske, V., \& Henning, T. 1999, A\&A, 349, 907

Mattioda, A. L., Allamandola, L. J., \& Hudgins, D. M. 2005a, ApJ, 629, 1183

Mattioda, A. L., Hudgins, D. M., \& Allamandola, L. J. 2005b, ApJ, 629, 1188

Meeus, G., Waters, L. B. F. M., Bouwman, J., et al. 2001, A\&A, 365, 476

Miyake, K., \& Nakagawa, Y. 1995, ApJ, 441, 361

Nomura, H., \& Millar, T. J. 2005, A\&A, 438, 923

Perrin, M. D., Duchêne, G., Kalas, P., \& Graham, J. R. 2006, ApJ, 645, 1272

Preibisch, T., Ossenkopf, V., Yorke, H. W., \& Henning, T. 1993, A\&A, 279, 577

Rettig, T., Brittain, S., Simon, T., et al. 2006, ApJ, 646, 342

Schräpler, R., \& Henning, T. 2004, ApJ, 614, 960

Siebenmorgen, R., Krügel, E., \& Mathis, J. S. 1992, A\&A, 266, 501

van Boekel, R., Waters, L. B. F. M., Dominik, C., et al. 2004, A\&A, 418, 177

van Boekel, R., Min, M., Waters, L. B. F. M., et al. 2005, A\&A, 437, 189

Verstraete, L., Puget, J. L., Falgarone, E., et al. 1996, A\&A, 315, L337

Visser, R., Geers, V. C., Dullemond, C. P., et al. 2007, ArXiv Astrophysics e-prints 